\title{
实物凭证标本作为命名动物新种必要性条件的挑 战:《国际动物命名法规》、物种保护与数码时代
}

\author{
陈 军 ${ }^{*}$ \\ (中国科学院动物研究所动物进化与系统学院重点实验室, 北京 100101)
}

\begin{abstract}
摘要: 随着濒危物种保护意识的不断提升和数字化技术的不断进步, 加之对 《国际动物命名法规》(第4版)相关条 款的不同理解, 近年来出现了不以保藏的死的动物个体实物标本为模式标本命名脊椎动物新种的案例, 引起了能 否不依据实物凭证标本命名动物新种的讨论。2015年Marshall和Evenhuis依据15张数码照片发表了昆虫一新种, 立 即引发了对这一论题的激烈争论。反对必须以实物标本作为模式标本者认为, 《国际动物命名法规》(第4版)第 73.1.4条款允许以图为正模来命名动物, 越来越多的“数码采集者”为依据图片发表新种提供了更大的可能, 而且 这样做可以避免因采集标本对濒危物种造成威胁; 坚持必须以实物标本作为模式标本者则认为, 《国际动物命名 法规》(第4版)十分强调以实物标本作为载名模式, 以图或其他非死的动物个体实物标本为依据发表新种可能因无 法获得更加详细、准确、全面的形态特征信息而造成学术上的缺憾或混乱, 也存在造假的可能性, 并且认为采集 标本并不是造成物种濒危或灭绝的原因。本文对上述观点进行了总结, 认为鉴于《国际动物命名法规》(第4版)中 存在模糊甚至相互矛盾的一些内容, 非常有必要进行一次全面的修订。在修订时, 应该考虑濒危物种保护、新技 术应用等诸多需求。鼓励依据实物标本命名动物新种, 在合理、适度、有序的原则下不断收集动物标本。
\end{abstract}

关键词: 实物凭证标本; 国际动物命名法规; 物种保护; 数码时代

\section{Challenges regarding the necessity of voucher specimens for naming a new animal species: the Code, species conservation and the digital age}

\author{
Jun Chen* \\ Key Laboratory of Zoological Systematics and Evolution, Institute of Zoology, Chinese Academy of Sciences, Beijing \\ 100101
}

\begin{abstract}
With increased awareness of endangered species conservation, the development of digital technology, and different interpretations of some articles in the International Code of Zoological Nomenclature (Fourth edition), in recent years some new vertebrate species have been named and published without preserved dead bodies as type specimens. This has triggered discussions on the necessity of voucher specimens for naming a new animal species. In 2015, after Marshall \& Evenhuis described a new insect species on the basis of 15 photographs only, a hot debate on this topic resulted. Supporters who advocate for naming new species without voucher specimens argue that, to name a new species without a voucher specimen is Code-compliant according to the Article 73.1.4. In addition, more and more skilled "digital collectors” would increase the likelihood of this kind of practice, and thus the threat to endangered species from collecting specimens could be reduced. Scholars on the other side of the debate argue that, in the Code, it is stressed that the specimen is the name-bearing type and not the illustration or description itself, and that describing new species based only on a picture and other non-physical specimens would result in errors in follow-up studies due to the lack of more detailed, accurate, and comprehensive morphological characteristics, along with possibilities of falsification. As well, collecting specimens is not the reason that species are endangered or extinct. In this article, the author summarizes the viewpoints of both sides of the debate. In consideration
\end{abstract}

收稿日期: 2017-09-17; 接受日期: 2017-11-15

基金项目: 国家自然科学基金(31372155)、科技部科技基础性工作专项(2014FY210200)、科技部国家国际科技合作专项(2015DFR30790)和国家科技基 础条件平台工作重点项目(2005DKA21402)

* 通讯作者 Author for correspondence. E-mail: chenj@ioz.ac.cn 
of some articles with ambiguous and even contradictory meanings, the author suggests that the International Code of Zoological Nomenclature (Fourth edition) be revised in response to the needs of endangered species conservation and new technological applications. Naming new species with voucher specimens should be encouraged, as well as collecting specimens using reasonable, moderate, and orderly principles.

Key words: voucher specimen; International Code of Zoological Nomenclature; species conservation; digital age

260多年前, 林奈将双名法引入生物分类学研 究, 极大地推动了人类对生物物种分类的认识, 掀 起了发现、命名新物种的热潮。但由于缺少相应的 规定, 很多学者依据自己的知识和经验, 采用各自 的标准和方法对物种命名和描述，由此产生了很多 混乱，导致出现了“第一次生物信息危机”(The first bioinformatics crisis) (Godfray, 2002)。也正是这些混 乱现象, 促使研究者开始重视分类学规范的制定, 并起草了一些规范物种命名的标准, 在此基础上, 逐渐形成了包括《国际动物命名法规》在内的一系 列生物命名法规。

第一版《国际动物命名法规》于1961年正式实 施, 以后随着时间的推移, 对其进行了3次修订。目 前执行的第四版于1999年出版，2000年1月1日起实 施。在国际动物命名法规中, 非常重视对模式标本 的指定、发表和保藏。第四版《国际动物命名法规》 在“第4章: 可用性的标准”中, 新增加“第16条: 1999 年以后发表的名称”, 对发表种级类群(新种和新亚 种)名称，规定 “载名模式的固定须是明确的”, 即 “必须在原始出版物中伴有: 有关命名分类单元的 正模或群模的明确固定; 对于正模和群模为现生标 本的存放地点, 可由一个明确的叙述来表明它们将 被(或已被)存放在一个收藏处, 并指明该收藏处的 名称和地点 (Every new specific and subspecific name... must be accompanied in the original publication by the explicit fixation of a holotype, or syntypes, for the nominal taxon; where the holotype or syntypes are extant specimens, by a statement of intent that they will be (or are) deposited in a collection and a statement indicating the name and location of that collection”) (International Commission on Zoological Nomenclature, 1999; 卜文俊和郑乐怡(译), 2007)。

基于国际动物命名法规的规定, 动物分类学工 作者一直以模式标本作为命名新种的实物凭证标 本, 并认为模式标本是依据形态特征开展动物物种 鉴定的最根本的依据。高水平的分类学研究, 检视
模式标本是不可或缺的步骤。不断增加模式标本藏 量也成为了很多自然历史博物馆、标本馆追求的目 标, 甚至在一定程度上成为了这些机构引以为豪的 评价指标。

\section{1 对《国际动物命名法规》有关条款的争论}

随着人们对濒危物种保护意识的提高, 以及近 年来图像采集数字化、高分辨率、自动化技术的迅 猛发展, 依据动物实物标本才能进行物种命名的必 要性受到了怀疑和挑战。Smith等(1991)在报道索马 里1种鸟类新种(Laniarius liberates Smith, Arctander, Fjeldså \& Amir)时, 认为该物种处于濒危状态, 在 对活体进行研究并提取羽毛和血液、拍照后将其释 放, 将羽毛、血液和由羽毛获得的线粒体DNA细胞 色素 $b$ 基因序列作为模式标本。Jones等(2005)发表了 发现于坦桑尼亚南部的灵长类 1 新种Lophocebus kipunji Ehardt, Butynski, Jones \& Davenport, 其正模 为野外拍摄的一张数码照片中的一只雄性成体, 副 模为一张数码照片中的一只无法辨别性别的成体, 附以对录制的成体鸣声的分析。Jones等在发表新种 时, 指出当地该种的 2 个种群数量都很小, 出于保 护的考虑, 没有猎捕标本作为模式标本。他们的文 章发表后引起了争论, Timm等(2005) 以及Landry (2005)认为Jones等这样做不符合国际动物命名法规, 所发表的新种名称是一个“裸名”(nomen nudum)。而 国际动物命名法委员会执行秘书Polaszek等(2005) 却指出, Jones等在发表新种前, 多次与国际动物命 名法委员会秘书处和多位著名的分类学家进行过 讨论, 确保他们发表的新种是国际动物命名法规所 允许的。

以上事例均集中于种群个体数量较少的濒危 脊椎动物, 而且作者也都对未能依据国际动物命名 法规进行物种命名进行过讨论, 并提出了已经采取 的或可能的补救措施(非致死性的抽取血样及毛发; 如果将来活体死亡后，可制作为实物凭证标本长期 保藏等), 似乎是在濒危物种保护与必须以实物标 
本作为模式标本命名物种之间采取的折中办法, 虽 然引起了一些争论, 但并未引发普遍的讨论。

2015年, Marshall和Evenhuis依据在南非拍摄的 2 只雌性个体的15张数码照片, 发表了昆虫纲蜂蚈 科 1 新种 (Marleyimyia xylocopae Marshall \& Evenhuis), 并对是否必须依据实物凭证标本来命名 昆虫新种提出了自己的看法(Marshall \& Evenhuis, 2015)。一方面他们承认实物标本是描述物种的“黄 金标准”(gold standard), 保留了未来研究所需要的 一整套包括内、外部形态特征和遗传特征在内的物 种特征, 是获取生物多样性信息的宝库, 持续性地 采集标本是开展生物多样性研究应该优先考虑的; 但同时他们也指出, 在某些情况下实难获得或保存 实物模式标本, 而且采集和运输标本面临诸多限制, 随着越来越多的技术熟练的“数码采集者”(digital collectors)进行大量影像资料的拍摄, 学者们将会 不可避免地接受不依据死的、永久保存在标本馆的 实物标本命名的新分类单元的发表, 采集标本虽然 值得进行, 但并非必要。该文一经发表即引发了对 是否可以完全依据数码照片命名动物新种的激烈 争论(Amorim et al, 2016; Ceríaco et al, 2016; Cianferoni \& Bartolozzi, 2016; Löbl et al, 2016; Marshall \& Evenhuis, 2016; Pape et al, 2016; Santos et al, 2016; Dubois, 2017; Garraffoni \& Freitas, 2017; Gutiérrez \& Pine, 2017; Shatalkin \& Galinskaya, 2017)。

以上争论的一个核心焦点是对于《国际动物命 名法规》(第4版)第73.1.4条款的不同理解。该条款 是“第73条: 在原始出版物中固定的载名模式(正模 和群模)” 中对正模进行说明中的一类情况的规定: 指定一个单一标本的图为正模, 将作为对所绘标本 的指定来对待; 该标本不再存在或不能追寻的事实 本身, 不能使此项指定成为无效(Designation of an illustration of a single specimen as a holotype is to be treated as designation of the specimen illustrated; the fact that the specimen no longer exists or cannot be traced does not of itself invalidate the designation) (International Commission on Zoological Nomenclature, 1999; 卜文俊和郑乐怡(译), 2007)。反对必须以 实物标本作为模式标本者认为, 法规中这一条款明 确允许以照片(图)作为正模来命名新种(Polaszek et al, 2005; Donegon, 2008; Marshall \& Evenhuis, 2015, 2016; Pape et al, 2016); 坚持必须以实物标本作为
模式标本者则指出, 制定该条款的本意是为解决 1931年以前一些老旧文献中依据图来命名新种与 新版法规有关条款规定所产生的冲突(Dubois \& Nemésio, 2007; Ceríaco et al, 2016), 而且在第4版法 规中, “72.5: 作为载名模式的候选资格” 中对于是否 可将图片作为载名模式有明确的规定: 72.5.6: 在一 命名种级类群分类单元是基于一幅绘图或一篇描 述, 或基于一项对一绘图或描述的文献参考的事例 中, 载名模式即是被绘制的或被描述的标本(而非 图示或描述本身) (In the case of a nominal species-group taxon based on an illustration or description, or on a bibliographic reference to an illustration or description, the name-bearing type is the specimen or specimens illustrated or described (and not the illustration or description itself))(International Commission on Zoological Nomenclature, 1999; 卜文俊 和郑乐怡(译), 2007)。

Wakeham-Dawson等(2002)分析了不同阶段国 际动物命名法规的异同, 认为一个新种只有指定被 保藏起来的死标本作为种名载体才能被科学命名 是一种误解, 如果新种的描述是基于非实物标本凭 证, 如图片、科学家的笔记、新闻报道等, 就已经 能确保新种命名符合法规了。相应地, 当初进行绘 图、记述、拍照以及从其身体上取组织样用于分析 遗传信息所使用的动物个体, 即使它(们)的活体最 后被释放到自然环境中以后也许再也见不到, 它 (们)也都应该是该物种的模式标本。他们甚至乐观 地预测, 以后所有的物种都可以基于从活体身上取 得的组织样开展分子生物学分析的结果来进行描 记。由于该文3位作者中前2位的单位标注为国际动 物命名法委员会秘书处(Secretariat, International Commission on Zoological Nomenclature), 他们的上 述观点被认为代表了国际动物命名法委员会的观 点, 往往被视作支持不必以实物标本作为模式标本 的依据。

\section{2 不以实物模式标本命名动物新种存在的 优缺点}

除了上述争论, 学者们还就依据非妥善保藏的 死的个体实物标本命名新种的优缺点进行了讨论。 可归纳如下:

(1)标本采集对物种灭绝的威胁。从人们对物种 
濒危原因以及保护措施的认识来看, 对于已确认种 群个体数量小、分布区域相互隔离的物种, 杀死其 活体作为模式标本, 确实有可能增加其灭绝的风 险。在这一问题上目前尚缺少说服力强的具体实 例。有学者以19世纪中叶灭绝的大海雀(Pinguinus impennis (Linnaeus, 1758)) 和20世纪中叶灭绝的墨 西哥姬鸮(Micrathene whitneyi graysoni (Ridgway, 1886))作为案例来说明标本采集是导致其灭绝的主 要原因(Minteer et al, 2014)。但也有学者指出, 目前 在全球标本收藏机构中保藏的102号大海雀标本中, 许多是在该物种灭绝以后才收集到的骨骼标本, 造 成该物种灭绝的主要原因是数千年来人类为获取 食物、油脂、羽毛而进行的猎杀; 对于墨西哥姬鸮, 采集信息显示, 目前全球收藏的9号标本是在该物 种个体非常常见的时期(1896-1932年)采集的, 其后 来灭绝的主要原因是栖息地被破坏以及外来物种 入侵(Rocha et al, 2014)。即便如此, 在报道一些明显 或推测处于濒危的脊椎动物新种时, 人们还是避免 捕杀活体作为模式标本。

除了上述Jones等(2005)以数码照片和鸣声录音 发表灵长类1新种外, 类似的报道还有: Thalmann和 Geissmann (2005)依据1991年在马达加斯加捕捉1只 活体后拍摄的照片、录像、鸣声录音以及采集的毛 发, 发表了灵长类1新种(Avahi cleesei Thalmann \& Geissmann); Mendes Pontes等(2006)在发表巴西灵 长类1新种(Cebus queirozi Mendes Pontes \& Malta) 时, 捕捉了 1 只活体认定其为正模, 进行测量、拍照 后将其释放, 并将在野外拍摄的1张照片中的1只性 别未知的亚成体作为副模; Gentile和Snell (2009)发 表发现于加拉帕戈斯群岛 1 个镾虲新种(Conolophus marthae Gentile \& Snell)时, 指定1只自由生活的雄 性成体作为正模, 在对其进行形态特征观测、抽取 血样、拍照和录像后, 在其后足皮下植入一个射频 识别装置; 2015年, 我国学者李成等以数码相片中 的1只雄性成体为正模, 2 只雌性成体为副模, 发表 了发现于我国西藏墨脱的灵长类1新种 (Macaca leucogenys Li, Zhao \& Fan) (Li et al, 2015)。

目前以濒危为理由而不杀死活体作为模式标 本的仅见于哺乳类、鸟类和爬行类, 对于这些经过 调查证明已处于濒危状态的新物种, 如何处理其发 表的新种名称, 有待国际动物命名法委员会进行商 讨, 并在新版法规中作出明确的规定。此外, 在以
图片为模式记录载体的情况下, 可以尽量采取一些 非致死性手段采集该物种个体的一些记录分类学 特征的证据, 例如鸣声、血样、毛发、粪便等, 分 析并描述其鉴别特征, 为以后物种的鉴定提供更多 的佐证。如果有可能, 可在该物种个体(最好是模式 标本的个体)死亡后, 及时将其制作成模式标本长 久保藏。

(2)依据非实物凭证命名物种存在的不足。依据 图片等非实物凭证命名动物新种或证明动物在某 地存在分布的一个无法回避的问题是造假。2008年 引起社会广泛关注的 “华南虎事件”已被证明造假 (http://society.people.com.cn/GB/8217/106495)。1998 年有学者依据实物报道了在印度尼西亚苏拉威西 岛北部海域发现腔棘鱼(coelacanth) (Erdmann et al, 1998), 在2000年有学者投稿给Nature 期刊, 以照片 为据声称他们早在1995年就在距该地 $2,000 \mathrm{~km}$ 的印 度尼西亚爪哇岛西南海域发现了腔棘鱼, 但经过对 比，他们提供的照片被证实与1998年报道中依据实 物拍摄的照片是同一张照片, 但做了细微的修改 (McCabe \& Wright, 2000)。虽然实物标本也存在造 假的可能性, 但相对于照片而言, 还是更容易辨 别。目前尚未发现依据造假图片发表动物新种的案 例, 但这方面的担心不无道理。

指定圈养的动物活体作为模式标本发表新种 也存在一定的不足。首先, 指定的活体动物有可能 逃逸, 从而失去实物标本; 其次, 活体死亡后如果 未能及时被发现，或者未能采取有效的保藏措施， 也会导致无法获得高质量的实物标本(Dubois \& Nemésio, 2007)。

此外, 仅仅依据非实物凭证发表新种, 有可能 因无法获得更加详细、准确、全面的形态特征信息 而造成学术上的缺憾或混乱。Jones等(2005)发表新 种Lophocebus kipunji后, 模式产地农民设置的陷阱 误杀了1只雄性亚成体, 其皮张、头骨、部分肢骨被 送往美国Field Museum of Natural History保藏。 Davenport等(2006)对其开展更加详细的分子系统学 分析后, 结合其头骨形态特征, 认为Jones等发表的 新种代表着1个新属Rungwecebus Davenport, Stanley, Sargis, De Luca Mpunga, Machaga \& Olson, 并 依据此标本, 结合Jones等(2005)的描述对该属特征 进行了界定。Robb等(2013)依据数码照片以及对鸣 声分析, 报道了阿曼东北部的鸟类 1 新种 (Strix 
omanensis Robb, van den Berg \& Constantine), 但 Kirwan等(2015)在核对保藏于世界各地的标本(包 括正模标本)并分析形态特征及开展分子生物学研 究后, 认为广泛报道分布于中东地区的Strix butleri (Hume, 1878)应该包括2个种, 并将另外1种作为新 种(Strix hadorami Kirwan, Schweizer \& Copete)予以 报道。虽然他们通过比较Strix omanensis的照片, 认 为它与Strix butleri比较近似, 但由于缺少实物标本, 无法进一步判断其分类地位。后来Robb等(2016)在 Strix omanensis的模式产地捕到1只活体, 对其进 行外部形态特征测量, 获取羽毛和血样, 拍照和录 音后将其释放, 通过形态特征分析和分子生物学研 究, 确认Strix omanensis 为Strix butleri的次级同物 异名。

对于无脊椎动物(包括昆虫)而言, 由于大多数 种类个体较小, 在野外拍摄的照片更难完整记录其 外部形态特征, 如果仅凭照片命名新种, 很有可能 会为以后的研究造成麻烦。

\section{3 数字化技术对发现新物种的帮助}

在坚持以实物凭证标本命名动物新种的同时, 也不可忽视数字化技术对发现新物种的帮助。为了 提高标本利用价值, 方便使用, 自20世纪中后期一 些机构相继开始了全球生物多样性数据平台建设, 并向社会开放。截止到2016年8月, 全球主要的生物 多样性信息网站(如GBIF、BioCASe、SpeciesLink) 已经拥有了 6.5 亿条信息, 其中 1.25 亿条来自收藏于 全球各地的实物标本信息(Güntsch et al, 2017)。我 国自2003年开始构建国家标本平台 (http://www. nsii.org.cn), 目前已有植物标本信息 992 万余条, 动 物标本信息343万余条。这些通过互联网共享的标 本信息为鉴定物种提供了更加高效、快捷的途径。

此外, 数码采集者拍摄的影像也为科研人员发 现动物新物种提供了非常有用的参考资料。2015年 克罗地亚的大学生Josip Skejo在Facebook “Orthoptera” (直翅目)群中发现有人上传了拍摄于菲律宾的 1张菱蝗科昆虫的照片, 经过与其他学者讨论, Skejo认为这是单型属Arulenus Stål, 1877中的1个未被 报道的物种。在其他同行的帮助下, 他从网上购买 到2头采自与拍摄照片同一海岛的雄性标本进行进 一步的形态观察, 同时与菲律宾的同行联系, 指导 他们对该种的生境进行观察和拍照, 并采集标本。
最终, Skejo与菲律宾的一位大学生合作者依据实物 标本, 共同发表了该新种(Arulenus miae Skejo \& Caballero), 并对该种的生态学特征进行了报道 (Skejo \& Caballero, 2016)。

\section{4 结语}

综上所述, 鉴于《国际动物命名法规》(第4版) 中存在一些模糊甚至相互矛盾的内容, 非常有必要 进行一次全面的修订。在修订时, 应该考虑濒危物 种保护、新技术应用等诸多因素。除对传统的以经 过处理制作且长期妥善保藏的动物身体全部或部 分作为新种命名载体外, 还应考虑借助于新技术探 讨物种鉴定的实物材料(如组织样、毛发、蜕皮、粪 便等)、通过载体记录的非实物材料(如鸣声、照片、 录像等) 以及对这些材料研究后得出的显示物种特 征的结果(如遗传物质序列、鸣声分析图表、内外部 形态结构三维图像等)在作为新种命名载体中的作 用和地位。

对于确实因为个体数量少, 或者暂时无法获得 实物标本作为模式标本, 但又能确认其为新种, 在 新版国际动物命名法规颁布实施之前, 可将其作为 已知属的未定种来对待。

对于未处于濒危状态的动物, 特别是个体较小 的无脊椎动物(包括昆虫), 则应坚持以死的、永久保 存在标本馆的实物标本作为模式标本来命名新种。 虽然遗传物质序列可以在物种鉴定方面发挥重要 的作用, 但对于物种而言, 遗传物质序列只是该物 种的基因型(genotype), 无法直接反映其全面的表 现型(phenotype)。当然, 在新的命名法规中, 应该鼓励 辅以遗传信息和其他非实物凭证信息来命名新种。

从生物学研究的角度出发, 更应鼓励依据实物 标本命名动物新种, 鼓励在合理、有序、适度的原 则下不断收集动物标本。因为实物标本不但携带了 分类鉴定、地理分布、采集人、采集时间等基本信 息, 而且包含了生态学、解剖学、遗传学以及一些 其他的附属信息, 这些信息在开展生物多样性研究 中价值巨大。正如Rocha等(2014)所说, 实物凭证标 本对于生物学研究的意义难以估计, 毕竟采集者无 法预料标本将来会被如何利用。

致谢: 在本文撰写过程中得到中国科学院植物研究 
所马克平研究员的鼓励, 特此致谢! 感谢两位匿名 审稿人和本文编辑对文稿提出重要的修改意见和 建议!

\section{参考文献:}

Amorim DS, Santos CMD, Krell FT, with other 47 co-authors (2016) Timeless standards for species delimitation. Zootaxa, 4137, 121-128.

Ceríaco LMP, Gutiérrez EE, Dubois A, supported by 490 signatories (2016) Photography-based taxonomy is inadequate, unnecessary, and potentially harmful for biological sciences. Zootaxa, 4196, 435-445.

Cianferoni F, Bartolozzi L (2016) Warning: potential problems for taxonomy on the horizon? Zootaxa, 4139, 128-130.

Davenport TRB, Stanley WT, Sargis EJ, De Luca DW, Mpunga NE, Machaga SJ, Olson LE (2006) A new genus of African monkey, Rungwecebus: morphology, ecology, and molecular phylogenetics. Science, 312, 1378-1381.

Donegon TM (2008) New species and subspecies descriptions do not and should not always require a dead type specimen. Zootaxa, 1761, 37-48.

Dubois A (2017) The need for reference specimens in zoological taxonomy and nomenclature. Bionomina, 12, 4-38.

Dubois A, Nemésio A (2007) Does nomenclatural availability of nomina of new species or subspecies require the deposition of vouchers in collections? Zootaxa, 1409, 1-22.

Erdmann MV, Caldwell RL, Moosa MK (1998) Indonesian 'king of the sea' discovered. Nature, 395, 335.

Garraffoni ARS, Freitas AVL (2017) Photos belong in the taxonomic Code. Science, 355, 805.

Gentile G, Snell H (2009) Conolophus marthae sp. nov. (Squamata, Iguanidae), a new species of land iguana from the Galápagos Archipelago. Zootaxa, 2201, 1-10.

Godfray HCJ (2002) Challenges for taxonomy. Nature, 417, 17-19.

Güntsch A, Hyam R, Hagedorn G, Chagnoux S, Röpert D, Casino A, Droege G, Glöckler F, Gödderz K, Groom Q, Hoffmann J, Holleman A, Kempa M, Koivula H, Marhold K, Nicolson N, Smith VS, Triebel D (2017) Actionable, long-term stable and semantic web compatible identifiers for access to biological collection objects. Database, Vol. 2017: article ID bax003; doi:10.1093/database/bax003.

Gutiérrez EE, Pine RH (2017) Specimen collection crucial to taxonomy. Science, 355, 1275.

International Commission on Zoological Nomenclature (1999) International Code of Zoological Nomenclature. 4th edn. International Trust for Zoological Nomenclature, London. http://www.iczn.org/iczn/index.jsp (accessed on 2017-08-21).

International Commission on Zoological Nomenclature (translated by $\mathrm{Bu}$ WJ, Zheng LY) (2007) International Code of Zoological Nomenclature, 4th edn. Adopted by the Interna- tional Union of Biological Sciences. Science Press, Beijing. (in Chinese). [卜文俊, 郑乐怡 (译) (2007) 国际动物命名 法规(第四版)/国际生物科学协会通过. 科学出版社, 北 京.]

Jones T, Ehardt CL, Butynski TM, Davenport TRB, Mpunga NE, Machaga SJ, De Luca DW (2005) The highland mangabey Lophocebus kipunji: a new species of African monkey. Science, 308, 1161-1164.

Kirwan GM, Schweizer M, Copete JL (2015) Multiple lines of evidence confirm that Hume's Owl Strix butleri (A. O. Hume, 1878) is two species, with description of an unnamed species (Aves: Non-Passeriformes: Strigidae). Zootaxa, 3904, 28-50.

Landry SO (2005) What constitutes a proper description? Science, 309, 2164.

Li C, Zhao C, Fan PF (2015) White-cheeked macaque (Macaca leucogenys): a new macaque species from Medog, southeastern Tibet. American Journal of Primatology, 77, 753-766.

Löbl I, Cibois A, Landry B (2016) Describing new species in the absence of sampled specimens: a taxonomist's own-goal. Bulletin of Zoological Nomenclature, 73, 83-86.

Marshall SA, Evenhuis NL (2015) New species without dead bodies: a case for photo-based descriptions, illustrated by a striking new species of Marleyimyia Hesse (Diptera, Bombyliidae) from South Africa. ZooKeys, 525, 117-127.

Marshall SA, Evenhuis NL (2016) Proxy types, taxonomic discretion, and taxonomic progress: a response to Löbl et al.. Bulletin of Zoological Nomenclature, 73, 87-92.

McCabe H, Wright J (2000) Tangled tale of a lost, stolen and disputed coelacanth. Nature, 406, 114.

Mendes Pontes AR, Malta A, Asfora PH (2006) A new species of capuchin monkey, genus Cebus Erxleben (Cebidae, Primates): found at the very brink of extinction in the Pernambuco Endemism Centre. Zootaxa, 1200, 1-12.

Minteer BA, Collins JP, Love KE, Puschendorf R (2014) Avoiding (re)extinction. Science, 344, 260-261.

Pape T, supported by 34 signatories (2016) Species can be named from photos. Nature, 537, 307.

Polaszek A, Grubb P, Groves C, Ehardt CL, Butynski TM (2005) What constitutes a proper description? Response. Science, 309, 2164-2166.

Robb MS, Sangster G, Aliabadian M, van den Berg AB, Constantine M, Irestedt M, Khani A, Musavi SB, Nunes JMG, Willson MA, Walsh AJ (2016) The rediscovery of Strix butleri (Hume, 1878) in Oman and Iran, with molecular resolution of the identity of Strix omanensis Robb, van den Berg and Constantine, 2013. Avian Research, 7, doi: 10.1186/s40657-016-0043-4.

Robb MS, van den Berg AB, Constantine M (2013) A new species of Strix owl from Oman. Dutch Birding, 35, 275-310.

Rocha LA, Aleixo A, Allen G, with other 122 co-authors (2014) Specimen collection: an essential tool. Science, 344, 
814-815.

Santos CMD, Amorim DS, Klassa B, Fachin DA, Nihei SS, de Carvalho CJB, Falaschi RL, Mello-Patiu CA, Couri MS, Oliveira SS, Silva VC, Ribeiro GC, Capellari RS, Lamas CJE (2016) On typeless species and the perils of fast taxonomy. Systematic Entomology, 41, 511-515.

Shatalkin AI, Galinskaya TV (2017) A commentary on the practice of using the so-called typeless species. ZooKeys, 693, 129-139.

Skejo J, Caballero JHS (2016) A hidden pygmy devil from the Philippines: Arulenus miae sp. nov. - a new species serendipitously discovered in an amateur Facebook post (Tetrigidae: Discotettiginae). Zootaxa, 4067, 383-393.
Smith EFG, Arctander P, Fjeldså J, Amir OG (1991) A new species of shrike (Laniidae: Laniarius) from Somalia, verified by DNA sequence data from the only known individual. Ibis, 133, 227-235.

Timm RM, Ramey RR II, The Nomenclature Committee of the American Society of Mammalogists (2005) What constitutes a proper description? Science, 309, 2163-2164.

Wakeham-Dawson A, Morris S, Tubbs P (2002) Type specimens: dead or alive? Bulletin of Zoological Nomenclature, 59, 282-284.

(责任编委: 卜文俊 责任编辑: 时意专) 